\title{
Magnetic field diffusion in ferromagnetic materials: fractional calculus approaches
}

\author{
Jordan Hristov $^{*}$ \\ Department of Chemical Engineering, University of Chemical Technology and Metallurgy,Bulgaria \\ jordan.hristov@mail.bg
}

\section{ARTICLE INFO}

\section{Article History:}

Received 29 March 2021

Accepted 24 July 2021

Available 17 August 2021

\section{Keywords:}

Magnetic field

Diffusion approximation

Fractional calculus

Integral method

Memory kernel effect

AMS Classification 2010:

35K57; 44Axx; $26 A 33$

\section{ABSTRACT}

The paper addresses diffusion approximations of magnetic field penetration of ferromagnetic materials with emphasis on fractional calculus applications and relevant approximate solutions. Examples with applications of time-fractional semi-derivatives and singular kernel models (Caputo time fractional operator) in cases of field independent and field-dependent magnetic diffusivities have been developed: Dirichlet problems and time-dependent boundary condition (power-law ramp). Approximate solutions in all theses case have been developed by applications of the integral-balance method and assumed parabolic profile with unspecified exponents. Tow version of the integral method have been successfully implemented: SDIM (single integration applicable to timefractional semi-derivative model) and DIM (double-integration model to fractionalized singular memory models). The fading memory approach in the sense of the causality concept and memory kernel effect on the model constructions have been discussed.

\section{Introduction}

There are many natural phenomena which can be modelled in diffusion approximations. Here magnetic field diffusions in solid ferromagnetics is considered with attempts to apply approximate solution based on synergism of fractional calculus and the integral-balance method in different versions. The main idea is to demonstrate the feasibility of both the fractional calculus approach and the integral solution.

In the context of the main idea of this communication magnetic diffusion of a field with parallel lines (see Figure 1) is taken as example. Two basic cases considering filed-independent and fielddependent diffusions with fixed (Dirichlet) and time dependent (power-law) boundary conditions are chosen as test examples. Moreover, the problem of magnetic field diffusion with memory is discussed with either the common time fractional operator of Caputo with singular kernel or from the more fundamental fading memory principle allowing different memory functions to be used.

\subsection{Magnetic field transport in conducting media}

The field transport in magnetizable and conducting media can be presented as superposition of the fundamental processes of advection and diffusion as key parts of describing behaviour of magnetic field in materials. In homogeneous (and ideal) materials, the magnetic field $\mathbf{B}$, the electric field $\mathbf{E}$ and the material velocity (mainly in the case of plasma) $\mathbf{v}$ are related by the following constitutive relationship [1]

$$
\mathbf{E}+\mathbf{v} \times \mathbf{B}=0
$$

It is worthy to mention, that if the material is not ideal,that is when the material resistance is finite then the right-hand side of (1) we have [1] 


$$
\mathbf{E}+\mathbf{v} \times \mathbf{B}=D_{\mu}(\nabla \times \mathbf{B})
$$

where $D_{\mu}=\sigma / \mu$ ( $\sigma$ is the material resistivity, $\mu$ is magnetic permeability) is the magnetic diffusivity. In such a case the magnetic field induction equation is [1]

$$
\frac{\partial \mathbf{B}}{\partial t}=\nabla \times(\mathbf{v} \times \mathbf{B})+\nabla \times\left(D_{\mu} \nabla \times \mathbf{B}\right)
$$

If a pure resistive magnetic diffusion is considered then Eq. (3) reduces to [1]

$$
\frac{\partial \mathbf{B}}{\partial t}=\nabla \times\left(D_{\mu} \nabla \times \mathbf{B}\right)
$$

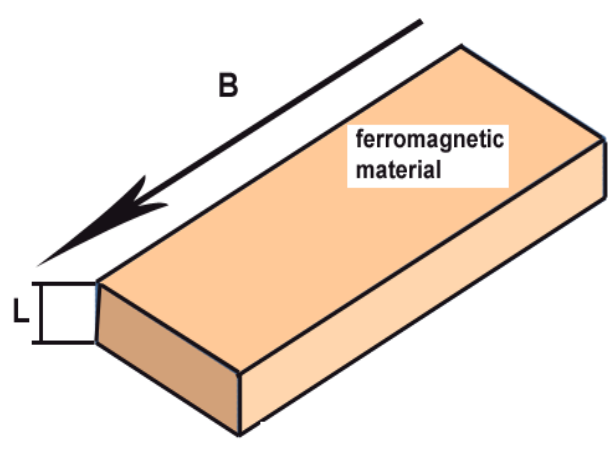

Figure 1. Schematic presentation of magnetic field with straight lines applied to a ferromagnetic material

The physics behind these relationships means that the changes in the magnetic field lines in time can be due to two principle causes: magnetic field advection (if the material is flowing as plasma or highly conductive fluid) and its diffusion through the material. Hence, as in the classical transport theory we assume a superposition of two transport mechanism: advection and diffusion. If the magnetic diffusivity $D_{\mu}$ is uniform (spatially independent), then it is possible to express (3), as [1]

$$
\frac{\partial \mathbf{B}}{\partial t}=\nabla \times(\mathbf{v} \times \mathbf{B})+D_{\mu} \nabla^{2} \mathbf{B}
$$

That is, the magnetic field flux velocity $\mathbf{w}$ is related to the temporal change of the induction $\mathbf{B}$ by the relation [1]

$$
\frac{\partial \mathbf{B}}{\partial t}=\nabla \times(\mathbf{w} \times \mathbf{B})
$$

and $\mathbf{w}$ is termed flux transporting velocity [1] In a particular case considered in this article if the pure resistive material is at issue, then $\mathbf{E}=$ $\eta \nabla \times \mathbf{B}$ and the ideal Ohm law holds (see (10) the magnetic flux velocity is practically equal to the velocity of the medium (flowing conductive medium) $\mathbf{v}$ and (10) becomes

$$
\mathbf{E}+\mathbf{v} \times \mathbf{B}=\nabla F
$$

and $F$ is an arbitrary function of integration [1]

\subsection{Magnetic field diffusion with straight field lines}

\subsubsection{Medium with field independent permeability}

If one-dimensional case is considered then equation (4) reduces to the following diffusion equation [1 5] with constant magnetic diffusivity.

$$
\frac{\partial B}{\partial t}=\frac{\partial}{\partial x}\left(D_{\mu} \frac{\partial B}{\partial x}\right)
$$

With uniform magnetic diffusivity $D_{\mu}=D_{\mu 0}=$ $\sigma / \mu=$ const. ( $\sigma$ is the resistivity of the material, $\mu=d B / d H=f(B)$ is the field dependent permeability of the material) and a sharp unit step at the boundary $x=0$ (Dirichlet problem), that is (i.e. for the case $\mu=d B / d H=f(B)=k_{B}=$ const.) we get

$$
B(x, 0)=\left\{\begin{array}{l}
+B_{0}, x>0 \\
-B_{0}, x<0
\end{array}\right.
$$

The case is relevant to an infinitesimally thin current sheet [1. If the field is maintained fixed at two boundary points of a finite domain $( \pm L)$ obeying the conditions $B(L, t)=-B(-L, t)=$ $B_{0}$, the solution of (8) with $D_{\mu}=D_{\mu 0}$ is [1]

$$
\begin{array}{r}
B(x, t)=\underbrace{B_{0} \frac{x}{L}}_{\begin{array}{c}
\text { stationary } \\
\text { profile }
\end{array}}+ \\
+\underbrace{2 \frac{B_{0}}{\pi} \sum_{k=1}^{\infty} \frac{1}{k} \exp \left[-k^{2} \pi^{2}\left(\frac{D_{\mu 0}}{L^{2}} t\right)\right] \sin \left(k \pi \frac{x}{L}\right)}_{\text {transient term }}
\end{array}
$$

The solution means very rapidly establishment of the magnetic field stationary profile $B_{0}(x / L)$. Moreover, taking into account the finite Ohmic heating $\left(j^{2} / \sigma\right)=\left(D_{\mu 0} / \mu\right)\left(B_{0} / L\right)^{2}$ per unit length of the medium due the continuous supply of magnetic energy through the boundaries with a rate $\left(D_{\mu 0} B_{0}^{2} / \mu L\right)[1]$ 


\subsubsection{Medium with field dependent permeability}

Commonly the power-law approximation [2] describes the magnetic field induction dependent on the field intensity, namely

$$
\bar{B}=\frac{B(H)}{B_{s}}=\left(\frac{H}{H_{s}}\right)^{\gamma}, \quad 0<\gamma<1
$$

where $B_{s}$ and $H_{S}$ are corresponding to the point of magnetic saturation (specific characteristics for every magnetic material that can be used as characteristic scales). Actually, this permits the diffusion equation to be presented in a dimensionless form as [2, 6]

$$
\frac{\partial \bar{B}}{\partial t}=D_{\mu}^{\bar{B}} \frac{\partial}{\partial x}\left(\bar{B}^{\beta} \frac{\partial \bar{B}}{\partial x}\right)
$$

where $\beta=\frac{1-\gamma}{\gamma}, D_{\mu}^{B}=\frac{\sigma}{\mu_{s} B_{s}^{\beta}}, \mu_{s}=\frac{B_{s}}{H_{s}}$.

Equation (12) is a degenerate parabolic equation because of the power-law diffusivity $D_{\mu}=D_{\mu}^{B} B^{\beta}$; in such a case the solution has a finite speed in contrast to model (8) where the solution speed is infinite. Hereafter, for the sake of simplicity of the expressions we will omit the symbol $\bar{B}$ and will use only $B$.

\subsection{Aim and motivation notes}

The following part of this article demonstrates how fractional calculus can be applied to solve magnetic diffusion models with either fieldindependent or field-dependent diffusivity. The assumption behind these models and the approximate solutions developed is there is no changes in the material resistivity (that is, no Joules effects as result of the magnetic field changes exist). The only magnetic field effect on the material property considered is the power-law dependence of the magnetic diffusivity as implicit performance of the field dependent magnetic permeability. The fractional calculus approach envisages two directions: 1) Semi-derivative approach to the parabolic model (8), and 2) Fractionalization of the magnetic diffusion equation through a constitutive flux-gradient relationship with singular memory. In addition, the general problem of the causality principle in modelling of non-local diffusion and the fading memory approach are discussed. In general, the models and the solutions developed consider the magnetic material as a semi-infinite with a boundary condition at $x=0$ since we are interested in the laws behind the magnetic field front propagation; before reaching the physical limit $L$ of the medium as in solution
(10). This approach allows straightforwardly seeing what would be the transient solution of the magnetic diffusion problem if memory formalism would be implemented in the diffusion model.

\subsection{Paper organization}

In what follows fractional semi-derivative diffusion model is developed by splitting the model (8) in section 2 and demonstrating two solutions with fixed (Dirichlet) (section 2.1.1) and timeramp boundary condition (section 2.1.2). Further, time-fractional models of magnetic diffusions are developed (section 3) through a constitutive equation with singular memory (section 3.1) with two problem solved (section 4): Dirichlet problem (section 4.2.1) and ramp (powerlaw) time-dependent boundary condition (section 4.2.2) solved by application of the Doubleintegration Method (DIM) (section 4.1) in the general case of of field-independent magnetic diffusivity. The model counterparts with fielddependent magnetic diffusivity are solved in sections (4.3) by preliminary transform of the diffusion term in two cases: Dirichlet problem (section 4.3.1) and ramp boundary condition (section 4.3.2). The fading memory principle and the causality concept are discussed in sections (5) and 5.1.1, respectively, thus allowing to construct a more general model of magnetic diffusion (section 5.1.2) and a qualitative analysis of the different kernel functions on it (section 5.1.3).

\section{Fractional calculus to magnetic diffusion problem}

Here we address three principle problems :

- Fractional calculus solution by semiderivatives of the problem with constant magnetic diffusivity with fixed and timedependent boundary conditions

- Fractional models of magnetic diffusion with singular memories as counterparts of the integer-order models (8) and (12).

- Fractional models based on the causality principle and fading memory concept

\subsection{Fractional calculus solution by semi-derivatives: general approach}

Consider the model (8) which can be presented as product of two operators 


$$
\begin{array}{r}
\left(\frac{\partial^{1 / 2} B}{\partial t^{1 / 2}}-\sqrt{D_{\mu}} \frac{\partial B}{\partial x}\right) \times \\
\times\left(\frac{\partial^{1 / 2} B}{\partial t^{1 / 2}}+\sqrt{D_{\mu}} \frac{\partial B}{\partial x}\right)=0
\end{array}
$$

where

$$
\frac{\partial^{1 / 2} B}{\partial t^{1 / 2}}=\frac{1}{\Gamma(1 / 2)} \frac{d}{d t} \int_{0}^{u} \frac{B(x, t)}{\sqrt{t-u}} d u-\frac{B(x, 0)}{\sqrt{\pi t}}
$$

is a Riemann-Liouville derivative of order $1 / 2$. In (13) only the second term has a physical meaning [7, 8, . Hence, the time-fractional equivalent of (8) is 9 ]

$$
\begin{array}{r}
\frac{\partial^{1 / 2} B}{\partial t^{1 / 2}}=-\sqrt{D_{\mu}} \frac{\partial \theta}{\partial x} \Rightarrow \\
\frac{\partial^{1 / 2} B(0, t)}{\partial t^{1 / 2}}=-\sqrt{D_{\mu}} \frac{\partial B(0, t)}{\partial x}
\end{array}
$$

Applying the operator $D_{t}^{-1 / 2}$ to both sides of (15) we get (16)

$$
B(0, t)=-\sqrt{D_{\mu}} \frac{\partial^{-1 / 2}}{\partial t^{-1 / 2}}\left[\frac{\partial B(0, t)}{\partial x}\right]
$$

With initial condition $B(x, 0)=0$, applying a single integration with respect to the spatial coordinate $x$ and using the Leibniz rule for differentiation under the integral sign we get

$$
\frac{d}{d t} \int_{0}^{\delta} B(x, t) d x=\sqrt{D_{\mu}} \frac{\partial^{1 / 2} B(0, t)}{\partial t^{1 / 2}}
$$

The upper terminal of the integral in (17) defines a sharp front of magnetic field penetration into the medium with conditions (Goodman's boundary condition [10,11])

$$
B(\delta)=0, \quad \frac{\partial B}{\partial x}(\delta)=0
$$

Equation (17) is the principle equation of SemiDerivative Integral Method-single integration ( SDIM-1) [12. The exact solution of this problem (8) is well -known [13], namely

$$
B_{\text {exact }}=1-\operatorname{erf}(\eta / 2)
$$

where $\eta=x / \sqrt{D_{\mu} t}$ is the Boltzmann similarity variable. The approximate solution developed in this work applies an assumed general parabolic profile with unspecified exponent

$$
B_{a}=B_{s}(1-x / \delta)^{n}
$$

This assumed profile satisfies all boundary conditions (18) for any values of the exponent $n$ [11.

\subsubsection{SDIM solution: Dirichlet problem}

With the assumed parabolic profile (20) and applying the Goodman boundary conditions we get $B_{a}(0, t)=B_{s}=1$. Now, replacing $B(x, t)$ in the integral relation (17) by the approximate profile (20) the result is

$$
\frac{d}{d t} \int_{0}^{\delta}\left(1-\frac{x}{\delta}\right)^{n} d x=\sqrt{D_{\mu}} \frac{D^{1 / 2}}{\partial t^{1 / 2}} C
$$

The integration of (21) with the initial condition $\delta(t=0)=0$ yields

$$
\frac{1}{n+1} \frac{d \delta}{d t}=\sqrt{D_{\mu}} \frac{1}{\sqrt{\pi t}} \Rightarrow \delta_{B}=\sqrt{D_{\mu} t} \frac{2(n+1)}{\sqrt{\pi}}
$$

Hence the approximate distribution $B_{a}(x, t)$ of the magnetic field in the material is

$$
B_{a}(x, t)=B_{s}\left(1-\frac{x}{\sqrt{D_{\mu} t}} \frac{\sqrt{\pi}}{2(n+1)}\right)^{n}
$$

Hence, in terms if Boltzmann similarity variable $\eta=x / \sqrt{D_{\mu} t}$ the front is defined by the equality $\eta=2(n+1) / \sqrt{\pi}$ (i.e. when $\left.x=\delta_{B}\right)$ since at this point $B_{a}=0$. The optimal solution of this problem, i.e. solution with minimal mean squared error of approximation over the entire magnetic field penetration layer is $n_{\text {opt }}=2.248$ (similar problem was resolved in [12 ). Comparative numerical simulations are presented in Figure 2.

That is, the dimensionless penetration depth corresponding to the optimal solution is $\delta_{B} / \sqrt{D_{\mu} t}=\frac{2(n+1)}{\sqrt{\pi}} \approx 3.665$. Here $\sqrt{D_{\mu} t}$ plays a role of a length scale. Taking into account that $D_{\mu}=(\sigma / \mu)$ any Joule heating can change the magnetic diffusivity, as well changes in $\mu$ due to temperature effects on the material resistivity and magnetic permeability, correspondingly. 


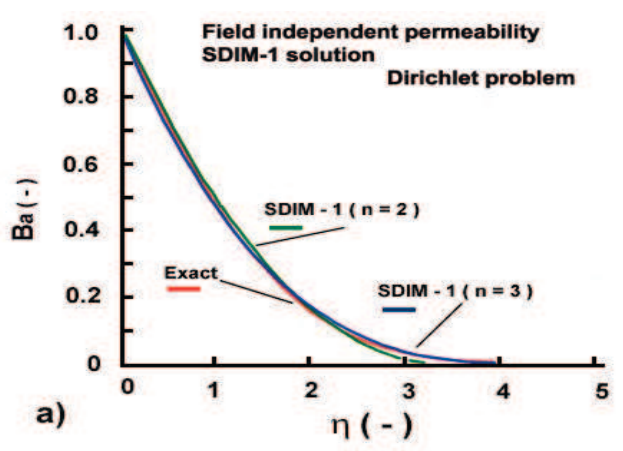

(a)

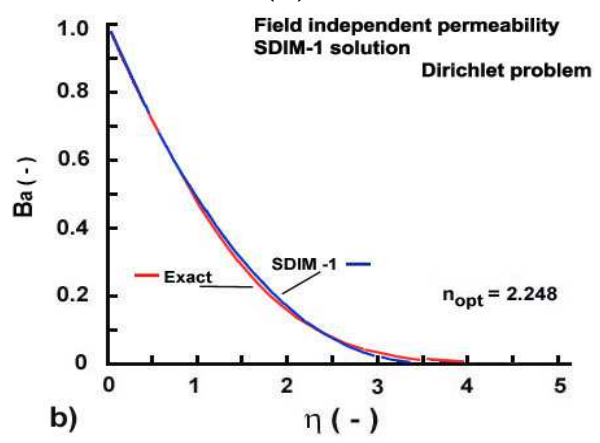

(b)

Figure 2. Approximate profiles developed by SDIM-1 approach and Dirichlet problem: for stipulated exponent $n=2$ (a) and optimal $n_{\text {opt }}=$ 2.248 (b), compared to exact solutions

\subsubsection{SDIM solution: Time-dependent boundary condition}

Let us consider a generalized ramp timedependent boundary condition $b_{0} t^{m / 2}$ with $m \geq$ 0 at $x=0$. This problem has an exact solution [13] (Chaptert 2 ) expressed through the error function (in terms of the process parameters discussed here), namely

$$
B_{e}=b_{0} \Gamma\left(\frac{m}{2}+1\right)(4 t)^{m / 2} i^{m} \Phi\left(\frac{x}{2 \sqrt{D_{\mu} t}}\right)
$$

which can be applied only by either numerical solution or tabulated data.

With the generalized parabolic profile (20) and the Goodman's boundary conditions we get

$$
\begin{aligned}
B_{a}(0, t)=B_{s}=b_{0} t^{m / 2}, \quad B_{a}(\delta)=B_{\infty} & =0, \\
\frac{\partial B_{a}}{\partial x}(x=\delta) & =0
\end{aligned}
$$

That is, the assumed profile is

$$
B_{a}=b_{0} t^{m / 2}\left(1-\frac{x}{\delta}\right)^{n}
$$

Now, applying the relation (17) the result is

$$
\frac{d}{d t} \int_{0}^{\delta} b_{0} t^{m / 2}\left(1-\frac{x}{\delta}\right)^{n} d x=\sqrt{D_{\mu}} \frac{D^{1 / 2}}{\partial t^{1 / 2}}\left(b_{0} t^{m / 2}\right)
$$

The integration of eq. (27) yields

$$
\begin{array}{r}
\frac{d}{d t}\left(b_{0} t^{m / 2} \frac{\delta}{n+1}\right)= \\
=\sqrt{D_{\mu}}\left[b_{0} \frac{\Gamma\left(\frac{m}{2}+1\right)}{\Gamma\left(\frac{m}{2}+\frac{1}{2}\right)} t^{m / 2-1 / 2}\right]
\end{array}
$$

The integration of (28) with the initial condition $\delta(t=0)=0$ yields

$$
\begin{array}{r}
\delta_{B}=\sqrt{D_{\mu} t} \frac{2(n+1)}{(m+1)} G_{m} \\
\Rightarrow \frac{\delta_{B}}{\sqrt{D_{\mu} t}}=C_{m(B)}^{n}=\frac{2(n+1)}{(m+1)} G_{m}
\end{array}
$$

where $G_{m}=\frac{\Gamma(m / 2+1)}{\Gamma(m / 2+1 / 2)}$ is a constant. Then, the approximate filed induction profile is

$$
\begin{aligned}
B_{a}(x, t)=b_{0} t^{m / 2} & \left(1-\frac{x}{\sqrt{D_{\mu} t} \frac{2(n+1)}{(m+1)} G_{m}}\right)^{n}= \\
& =b_{0} t^{m / 2}\left(1-\frac{\eta}{\frac{2(n+1)}{(m+1)} G_{m}}\right)^{n}
\end{aligned}
$$

Hence, the front is defined by the condition $\eta=$ $\frac{2(n+1)}{(m+1)} G_{m}$. The optimal values of the exponent $n$ depend on the rate of the surface magnetization, i,e. on the values of $m$. The minimization of the squared mean error of approximation for different values of $m$ yields optimal exponents summarized in Table 1. Plots of the approximate solutions are shown in Figure 3. 


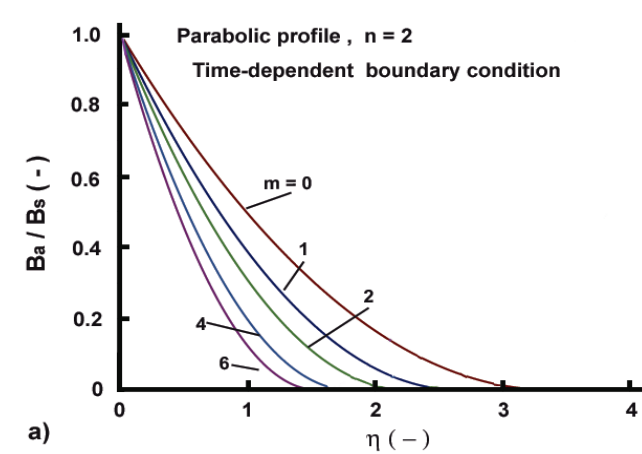

(a)

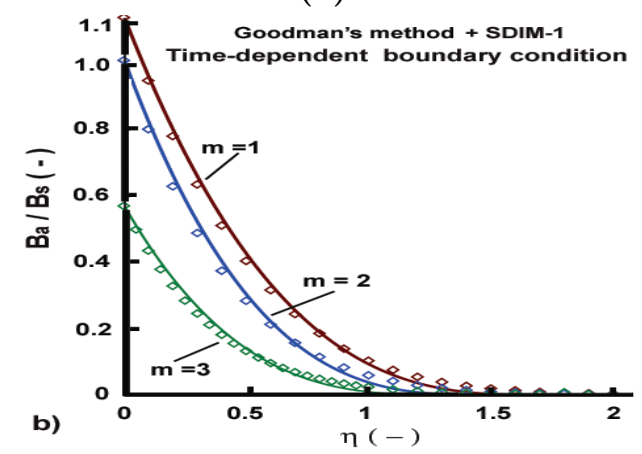

(b)

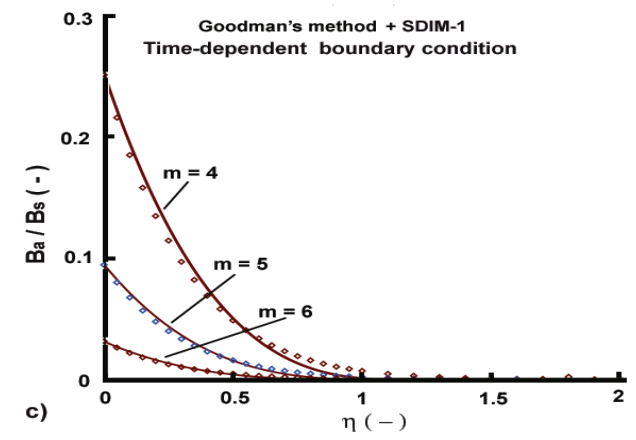

(c)

Figure 3. Normalized magnetic field profiles developed by SDIM-1 for different values of the parameter $m$ of the surface ramping magnetization: a) SDIM-1 solution with stipulated parabolic profile exponent $n=2 ; \mathrm{b}$, c) SDIM-1 solutions with optimal exponents: Comparison with exact solutions (tabulated) from 13.

Table 1. SDIM-1:Optimal exponents for different values of $m$

\begin{tabular}{lllllll}
$\mathbf{m}$ & $\mathbf{1}$ & $\mathbf{2}$ & $\mathbf{3}$ & $\mathbf{4}$ & $\mathbf{5}$ & $\mathbf{6}$ \\
\hline $\mathrm{n}$ (optimal) & 1.336 & 1.618 & 1.822 & 1.919 & 2.158 & 2.302
\end{tabular}

\section{Fractional models of magnetic diffusion: simplified approach}

Here we address magnetic diffusion equation with memory. Precisely, the memory function used to model is power-law with allows the fractional Caputo derivative to be applied.

\subsection{Magnetic flux with memory: general approach}

Let us consider a finite speed of the diffusion magnetic field into the material which cannot be assured by the parabolic model (8). In such a case following the causality principle that the reaction should follow the cause, a time shift between them can be presented through a convolution integral, that is

$$
\begin{aligned}
& j_{\mu}(x, t)=\underbrace{-D_{\mu} \nabla B(x, t)}_{\text {instantaneous (long times) }}- \\
& \underbrace{-D_{\mu_{1}} \int_{0}^{\infty} R(t-\tau) \nabla B(x, t-\tau) d \tau}_{\text {relaxation (memory ef fect) }}
\end{aligned}
$$

with a memory $R(t)$ controlled by a fractional parameter $\alpha, 0<\alpha<1$. In (31) the first term is relevant to long times where the relaxation disappears, known also as instantaneous term. If only this term is considered we get the parabolic model (8) with infinite speed of the solution. Now, we will omit this term in order to develop a model of magnetic diffusion of subdiffusion type. Applying the continuity equation

$$
\frac{\partial B}{\partial t}=-\frac{\partial}{\partial x} j_{\mu}
$$

as well as omitting the term $-D_{\mu} \nabla B(x, t)$ (and for the sake of simplicity getting $D_{\mu_{1}}=D_{\mu}$ ) we get a general relationship

$$
\frac{\partial B}{\partial t}=D_{\mu} \int_{0}^{\infty} R(t-\tau) \frac{\partial^{2} B(x, t-\tau)}{\partial x^{2}} d \tau
$$

The function of $R(t)$ depends strongly on the physics of the magnetization and the material properties itself.

\section{Fractional models of magnetic diffusion: Singular memory approach}

As first example we will address a singular power law memory. In such a case the memory integral in (33) becomes a Riemann-Liouville integral of order $\alpha$, namely 


$$
\begin{aligned}
I_{t}^{\alpha}= & \frac{1}{\Gamma(\alpha)} \int_{0}^{t}(t-\tau)^{\alpha-1} \frac{\partial^{2} B(x, \tau)}{\partial x^{2}} d \tau= \\
& =D_{t}^{-\alpha}\left[\frac{\partial^{2} B(x, \tau)}{\partial x^{2}}\right], \quad 0<\alpha<1
\end{aligned}
$$

and the flux-gradient relationship can be expressed as

$$
j_{\mu}=-D_{\mu} I_{t}^{\alpha}\left(\frac{\partial B}{\partial x}\right)
$$

Then, the application of the continuity equation yields

$$
\frac{\partial B}{\partial t}=D_{\mu} I_{t}^{\alpha}\left(\frac{\partial^{2} B}{\partial x^{2}}\right)=D_{\mu}\left[D_{t}^{-\alpha}\left(\frac{\partial^{2} B}{\partial x^{2}}\right)\right]
$$

Applying the operator $D_{t}^{\alpha-1}$ to both sides of (36) and recalling the semi group properties of the fractional derivatives and integrals (here we consider Caputo time-fractional derivative) we get

$$
\frac{\partial^{\alpha} B}{\partial t^{\alpha}}=D_{\mu} \frac{\partial^{2} B}{\partial x^{2}}
$$

which the well-known time-fractional diffusion (subdiffusion equation) with boundary and initial conditions

$$
\begin{array}{r}
B(0, t)=B_{s}(t), \quad t \geq 0, \\
B(x, 0)=B_{\infty}=0, \quad x>0
\end{array}
$$

Now, the magnetic diffusivity has a dimension $\left[D_{\mu}\right]=\left[\mathrm{m}^{2} / \mathrm{sec}^{\mu}\right]$. With $D_{\mu}$ independent of the time and space as well magnetic field independent, the linear problem is (37) with which the double integration method will be demonstrated next.

\subsection{Double integration method (DIM)}

The first step of DIM is the integration of (37) from 0 to $x$ [14]

$$
\int_{x}^{\delta} \frac{\partial^{\alpha} B}{\partial t^{\alpha}} d x=D_{\mu} \frac{\partial B(x, t)}{\partial x}-D_{\mu} \frac{\partial u(0, t)}{\partial x}
$$

Taking into account that the single integration from 0 to $\delta$ can be presented as a sum $\int_{0}^{\delta} f(x) d x=$

$$
\begin{aligned}
\int_{0}^{x} f(x) d x+ & \int_{x}^{\delta} f(x) d x \\
\text { obtain } & =-D_{\mu} \frac{\partial}{\partial x} f(x=0) \text { we can } \\
\int_{x}^{\delta} \frac{\partial^{\alpha} B}{\partial t^{\alpha}} d x & =-D_{\mu} \frac{\partial B(x, t)}{\partial x}
\end{aligned}
$$

The second step of DIM is the integration of (40) from 0 to $\delta$

$$
\int_{0}^{\delta}\left(\int_{x}^{\delta} \frac{\partial^{\alpha} B}{\partial t^{\alpha}} d x\right) d x=D_{\mu} B(0, t)
$$

Equation (41) is the principle relationship of the double integration method when the differential equation is of a fractional order [14]

\subsection{Field independent magnetic permeability}

Now, we will apply the integral-balance solutions to the time-fractional magnetic diffusion equation in two cases : fixed boundary condition (Dirichlet problem and time-ramping boundary condition (power-law).

\subsubsection{Dirichlet problem}

Now, we will apply DIM to (37) with assumed generalized parabolic profile. In this case we have

$$
\frac{\partial B_{a}(x, t)}{\partial t}=\frac{x}{\delta^{2}} n\left(1-\frac{x}{\delta}\right)^{n-1} \frac{d \delta}{d t}
$$

and incorporating this approximation in to the Caputo derivative one obtain

$$
\begin{array}{r}
\int_{0}^{\delta}\left[\int_{x}^{\delta}{ }_{C} D^{\alpha} B_{a}(x, t) d x\right] d x= \\
=\frac{1}{\Gamma(1-\alpha)} \int_{0}^{t} \frac{1}{(t-\tau)^{\alpha}} \frac{1}{(n+1)(n+2)} \frac{d \delta^{2}}{d t} d \tau
\end{array}
$$

$$
\begin{array}{r}
\int_{0}^{\delta}\left[\int_{x}^{\delta}{ }_{C} D_{t}^{\alpha} B_{a}(x, t) d x\right] d x=\frac{D_{t}^{\alpha}\left(\delta^{2}\right)}{N_{C}}, \\
N_{C}=(n+1)(n+2)
\end{array}
$$

$$
{ }_{C} D_{t}^{\alpha} \delta^{2}=D_{\mu}[(n+1)(n+2)]
$$


Therefore, the fractional integrations (with the physical condition $\delta(t=0)=0)$ results in

$$
{ }_{C} D_{t}^{\alpha} \delta(t)=\sqrt{D_{\mu} t^{\alpha}} \sqrt{\frac{(n+1)(n+2)}{\Gamma(1+\alpha)}}
$$

Therefore the approximate solution is

$$
\begin{gathered}
B_{a}=\left(1-\frac{x}{\sqrt{D_{\mu} t^{\alpha}} F_{n} j_{\alpha}}\right)^{n}, \\
F_{n}=\sqrt{(n+1)(n+2)}, \quad j_{\alpha}=1 / \sqrt{\Gamma(1+\alpha)}
\end{gathered}
$$

The solution defines a non-Boltzmann similarity $\eta_{\mu}=x / \sqrt{D_{\mu} t^{\alpha}}$. Numerical simulations are presented in Figure 4 . For more details related to the optimization of the solution and the technology of DIM to fractional subdiffusion models see the extended analysis in [14].

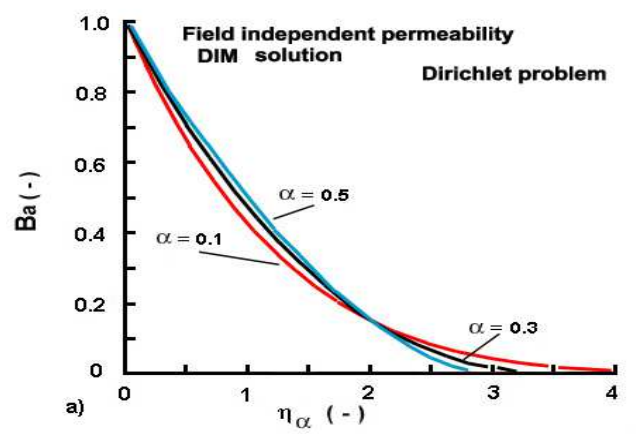

(a)

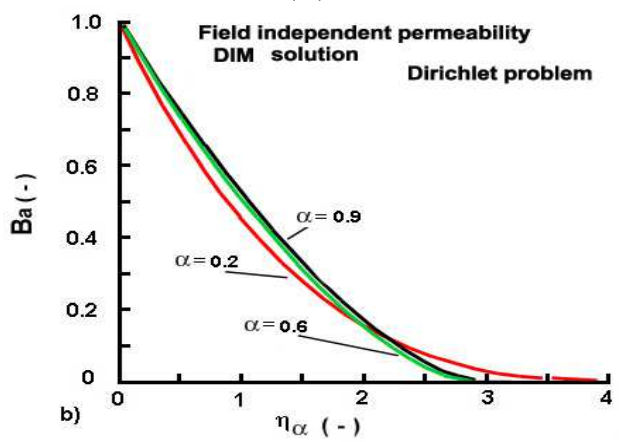

(b)

Figure 4. DIM solutions to the magnetization of field-independent material with Dirichlet Boundary condition, with : stipulated exponent of the parabolic profile $n=2$ (a) and optimal exponent $n_{\text {opt }}=2.248$ (b), compared to exact solutions

\subsubsection{Time-dependent boundary condition}

With time-dependent (power-law) boundary condition $B_{a}(0, t)=B_{s}=b_{0} t^{m / 2}$ the generalized parabolic profile (20) with the Goodman's boundary conditions (25) we get the assumed profile (26). Then, with the integral relation (41) we get

$$
\int_{0}^{\delta}\left(\int_{x}^{\delta} \frac{\partial^{\mu} B_{a}}{\partial t^{\mu}} d x\right) d x=D_{\mu} B(0, t)=D_{\mu} b_{0} t^{m / 2}
$$

With (42) incorporated in (48) we have

$$
\begin{array}{r}
\int_{0}^{\delta}\left(\int_{x}^{\delta} \frac{\partial^{\mu}}{\partial t^{\mu}}\left[\left(b_{0} t^{m / 2}\right) \times \frac{\partial B_{a}}{\partial t}\right] d x\right) d x= \\
=D_{\mu} b_{0} t^{m / 2}
\end{array}
$$

The integration in left-hand side of (49) yields

$$
\begin{aligned}
{ }_{C} D_{t}^{\mu}\left(\delta^{2} b_{0} t^{m / 2}\right) & =D_{\mu}\left(b_{0} t^{m / 2}\right) N_{C}, \\
N_{C} & =(n+1)(n+2)
\end{aligned}
$$

$$
\begin{aligned}
& \delta^{2} b_{0} t^{m / 2}=D_{\mu} N_{C} G_{m}^{\alpha} b_{0} t^{m / 2+\alpha}, \\
& G_{m}^{\alpha}=\left(\frac{\Gamma(m / 2+1)}{\Gamma(\alpha+m / 2+1)}\right) \\
& \delta^{2}=D_{\mu} t^{\alpha} N_{C} G_{m}^{\alpha} \Rightarrow \delta_{m}^{\alpha}=\sqrt{D_{\mu} t^{\alpha}} \sqrt{N_{C} G_{m}^{\alpha}}
\end{aligned}
$$

Hence, the approximate solution is

$$
B_{a, m}^{\alpha}=b_{0} t^{m / 2}\left(1-\frac{x}{\sqrt{D_{\mu} t^{\alpha}} \sqrt{N_{C}} \sqrt{G_{m}^{\alpha}}}\right)^{n}
$$

This solution defines a non-Boltzmann similarity variable $\eta_{\alpha}=x / \sqrt{D_{\mu} t^{\alpha}}$. Numerical simulations with various values of the fractional order $\alpha$ and the non-linear parameter $\beta$ are shown in Figure 5 . 


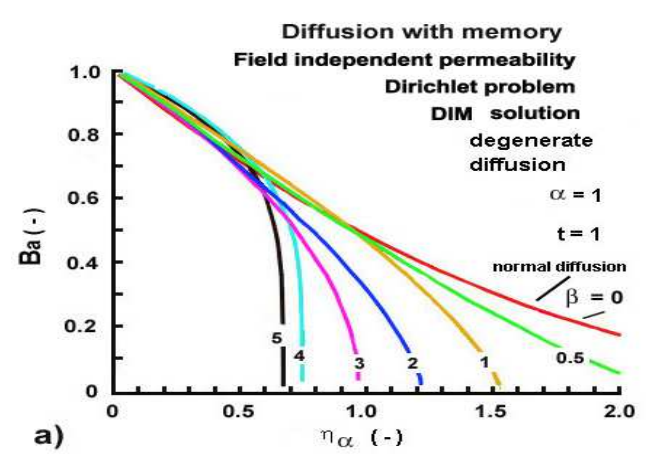

(a)

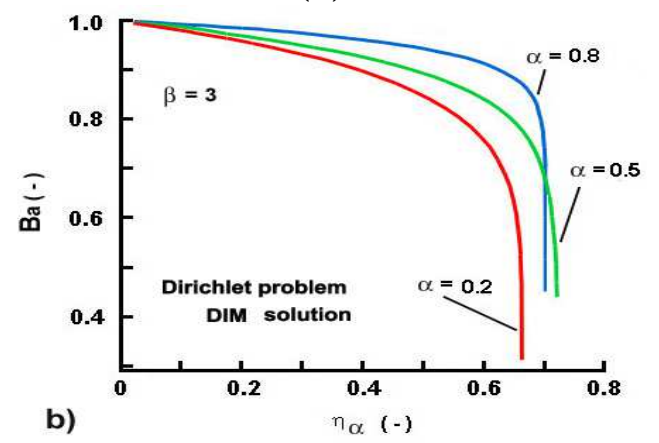

(b)

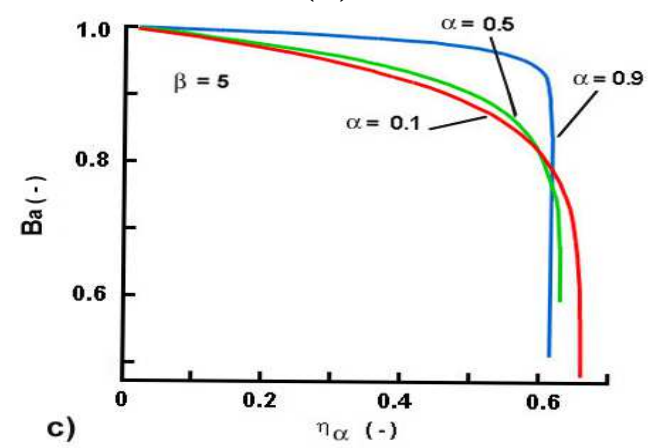

(c)

Figure 5. Approximate profiles for stipulated exponent $n=2$ (a) and optimal $n_{\text {opt }}=2.248$ (b) compared to exact solutions

\subsection{Field dependent magnetic permeability}

The application of the integral method needs a preliminary treatment of the of the diffusion term in the right-hand side of (12), namely [15, 16]

$$
D_{\mu}^{B} B^{m} \frac{\partial u}{\partial x}=\frac{D_{\mu}^{B}}{\beta+1} \frac{\partial B^{\beta+1}}{\partial x}
$$

Therefore, the result (53) can be considered as a non-linear counterpart of the constitutive equation (32), namely

$$
\frac{\partial B}{\partial t}=\frac{D_{\mu}^{B}}{\beta+1} \int_{0}^{\infty} R(t-\tau) \frac{\partial^{2} B^{\beta+1}(x, t-\tau)}{\partial x^{2}} d \tau
$$

With a singular (power-law) memory function, similarly to transformations done in 3.1 we get a fractional analogue of (37) with non-linear diffusion term (similar problem was solved in [16]).

$$
\frac{\partial^{\alpha} B}{\partial t^{\alpha}}=D_{\mu}^{B} \frac{1}{\beta+1} \frac{\partial^{2} B^{\beta+1}}{\partial x^{2}}
$$

Then applying DIM we have

$$
\int_{0}^{\delta} \int_{x}^{\delta} \frac{\partial^{\alpha} B(x, t)}{\partial t^{\alpha}} d x d x=\frac{D_{\mu}^{B}}{\beta+1} B^{\beta+1}(0, t)
$$

This is the principle DIM integral relationship when the diffusion term has a power-law nonlinearity.

\subsubsection{Dirichlet problem: Approximate solution}

With Caputo time-fractional derivative and the assumes parabolic profile (201) as well as by help of (41) the integration in LHS of Eq.(56) yields

$$
{ }_{C} D_{t}^{\alpha} \delta^{2}=D_{\mu}^{B} \frac{(n+1)(n+2)}{\beta+1}
$$

That is

$$
\delta^{2}=D_{\mu}^{B} \frac{N_{C}}{(\beta+1)} \frac{t^{\alpha}}{\Gamma(\alpha+1)}
$$

Hence, the penetration depth is

$$
\delta_{B}^{\alpha}=\sqrt{D_{\mu}^{B} t^{\alpha}} \sqrt{\frac{N_{C}}{(\beta+1)} \frac{1}{\Gamma(\alpha+1)}}
$$

and the approximate solution of (55) can be expressed as

$$
B_{a}(x, t)=\left(1-\frac{x}{\sqrt{D_{\mu}^{B} t^{\alpha}} \sqrt{\frac{(n+1)(n+2)}{(\beta+1) \Gamma(1+\alpha)}}}\right)^{n}
$$

The solution defines a new similarity variable $\eta_{\alpha}=x / \sqrt{D_{\mu}^{B} t^{\alpha}}$. For $\alpha=1$ it reduces to the classical Boltzmann similarity variable $\eta_{\alpha=1}=$ $x / \sqrt{D_{\mu}^{B t}}$. 


\subsubsection{Time-dependent boundary condition: Approximate solution}

From (56) it follows that the right-hand side is $\frac{D_{\mu}^{B}}{\beta+1} B^{\beta+1}(0, t)$. Then, if the boundary condition is of power law type $B_{s}=B(0, t)=b_{0} t^{m / 2}$ we have

$$
\frac{D_{\mu}^{B}}{\beta+1} B^{\beta+1}(0, t)=\frac{D_{\mu}^{B}}{\beta+1}\left(b_{0} t^{m / 2}\right)^{\beta+1}
$$

Therefore the DIM integral solutions is

$$
\int_{0}^{\delta} \int_{x}^{\delta} \frac{\partial^{\alpha} B(x, t)}{\partial t^{\alpha}} d x d x=\frac{D_{\mu}^{B}}{\beta+1}\left(b_{0} t^{m / 2}\right)^{\beta+1}
$$

Now, repeating the integration in the left-hand side of (62), as in (48) and (49) we get

$$
\begin{array}{r}
{ }_{C} D_{t}^{\mu}\left(\delta^{2} b_{0} t^{m / 2}\right)= \\
\frac{D_{\mu}^{B}}{\beta+1} b_{0}^{\beta+1} t^{m(\beta+1) / 2} N_{C}
\end{array}
$$

The fractional integration in (63) yields

$$
\begin{array}{r}
\delta^{2} b_{0} t^{m / 2}= \\
\frac{D_{\mu}^{B}}{\beta+1} G_{\alpha, \beta}^{m} N_{C} b_{0}^{\beta+1} t^{m(\beta+1) / 2+\alpha}
\end{array}
$$

where

$$
G_{\alpha, \beta}^{m}=\frac{\Gamma\left(\frac{m(\beta+1)}{2}+1\right)}{\Gamma\left(\alpha+\frac{m(\beta+1)}{2}+1\right)}
$$

The re-arrangement in (65) results in

$$
\delta^{2}=\frac{D_{\mu}^{B}}{\beta+1} G_{\alpha, \beta}^{m} N_{C} b_{0}^{\beta} t^{\left[\frac{m}{4}(\beta-1)+\alpha\right]}
$$

In a more useful form we have

$$
\delta=\sqrt{D_{\mu}^{B} t^{\frac{m(\beta-1)+4 \alpha}{4}}} \sqrt{\frac{G_{\alpha, \beta}^{m} N_{C} b_{0}^{\beta}}{\beta+1}}
$$

The exponent $\frac{m(\beta-1)+4 \alpha}{4}$ in (67) should be positive since we have to assure a positive growth of the front $\delta$. Therefore, the condition that should be obeyed is $\beta>1-4 \alpha / m$. Taking into account that $0<\alpha<1$ and $m=1,2,3 \ldots$, then the condition imposed on $\beta$ is satisfied.

To clarify this point, since $\beta=(1-\gamma) / \gamma$ where $0<\gamma<1(\gamma=0.22$ for steel [2] for example) we have always $\beta>1$. In the particular case with steel magnetization $(\gamma=0.22)$ we get $\beta=3.545$. In such a case the diffusion model (12) is a degenerate diffusion equation with convex solutions moving as almost sharp waves [15, 16]. In such a case the exponent of the parabolic profile (20) is $n=1 / \beta<1$ [15]. It is noteworthy to mention that that the parabolic profile (20) with $n<1$ generate convex profiles, while for $n>1$ the profiles are concave. Profile of approximate solutions showing competitive actions of the subdiffusion behaviour (trough the fractional parameter $\alpha$ ) and the diffusion non-linearity (through the exponent $\beta$ ) are shown in Figure 6

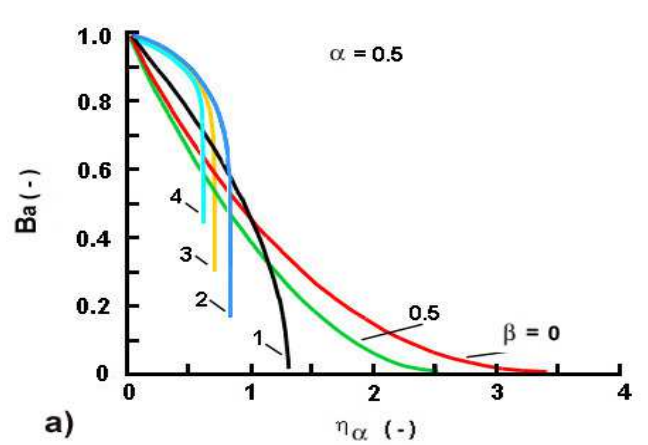

(a)

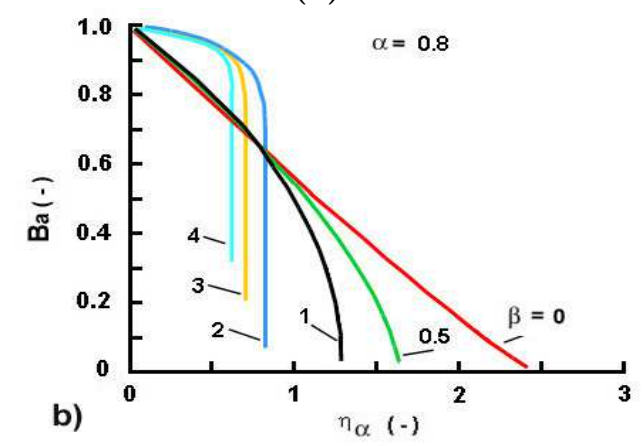

(b)

Figure 6. DIM solutions to the magnetization of field-dependent material with time-dependent boundary condition and optimal exponents [16] showing how the value of the exponent $\beta$ deforms the solution profile towards a rectangular wave with sharp front: a) Case with $\alpha=0.5$, b), case with $\alpha=0.8$ 


\section{Fractional models by fading memory approach}

\subsection{Fading memory principle}

For simple materials [17 21, the fading memory concept relating the flux to its gradient of a certain transported quantity $A$, is modelled by the following integro-differential equation

$$
\begin{array}{r}
j_{A}(x, t)=-D_{A 0} \frac{\partial A}{\partial x}(x, t)- \\
-D_{A 1} \int_{-\infty}^{t} R(t-\tau) \frac{\partial A}{\partial x}(x, \tau) d \tau
\end{array}
$$

This is the Boltzmann linear superposition functional [20] with a memory kernel $R(t, z)$. In (68) $D_{A 0}$ and $D_{A 1}$ are transport (diffusion) coefficients (diffusivities). In fact, we assume a linear superposition of two fluxes

$$
j_{A}(x, t)=\underbrace{j_{A 0}}_{\begin{array}{c}
\text { instantaneous } \\
\text { flux }
\end{array}}+\underbrace{j_{A 1}}_{\begin{array}{c}
\text { transient } \\
\text { flux with finite speed }
\end{array}}
$$

Actually, the convolution integral in (68) is Stieltjes integral but because there is a restriction imposed on $R(t, z)$ to be casual function, i.e. $R(t<0, z)=0$ we may set the lower terminal to $t=0$. Thus, the gradient of the flux $j_{A}$ can be presented in a general form as

$$
\begin{gathered}
\frac{\partial}{\partial x} j(x, t)=-D_{A 0} \frac{\partial^{2}}{\partial x^{2}} A(x, t)- \\
-D_{A 1} \int_{0}^{t} R(t-\tau) \frac{\partial^{2}}{\partial x^{2}} A(x, \tau) d \tau
\end{gathered}
$$

In (69) the first term is the long time, or instantaneous diffusion term, while the second is relevant to the finite sped of the diffusion wave of $A(x, t)$ . This is a general linear expression of the fading memory principle since the transport coefficients are constants.

If now the quantity $A$ is replaced by the magnetic field induction $B(x, t)$ we get the formulation (31). Moreover, if there is no flux relaxation and the speed is infinite, then the second term in (68) (as well as in (69) and (70)) is zero and the result is the classical $j_{A}(x, t)=-D_{A 0} \frac{\partial A}{\partial x}(x, t)$ which gets different names as Fick's (diffusion), Fourier (heat conduction) or Newton law (diffusion of momentum) laws.
The main idea behind the fading memory principle is to assure the causality of the models of dynamic systems (changing in time) as it is explained next

\subsubsection{Causality principle}

In all applied cases the chronological condition allows the causal relation to be satisfied (i.e. the time-shift between cause and effect) [22, i.e. always the cause precedes the effect. The principle conditions of the causality principle are [22]:

- Primitive causality: The effect cannot precede the cause.

- Relativistic causality: No signal can propagate with velocity greater than the speed of the light in the vacuum. It could be considered as a macroscopic causality condition.

Further, the causality concept means that the functions describing transients should be: vanishing over a range of values of its arguments (as the memory functions in the convolution integrals).

If we consider the physical system of the magnetic field diffusion with a time-dependent cause) $B_{s}(t)$ and the corresponding effect $B(x, t)$ the following conditions are obeyed [22].

C1: Linearity. This corresponds to the superposition principle in its simple version implying that the output is a linear functional of the input

$$
B(t)=\int_{-\infty}^{\infty} R(t, \tau) B_{s}(\tau) d \tau
$$

C2: Time-translation invariance. In this case the linear functional can be expressed as

$$
B(x, t)=\int_{-\infty}^{\infty} R(t-\tau) B_{s}(\tau) d \tau=R(t) * B_{s}(t)
$$

C3: Primitive causality condition. The input cannot precede the output. As consequence, $R(\tau)$ should be a causal function. Moreover, this is equivalent to setting the lower terminal in the (71) and (72) equal to zero, as mentioned in preceding point related to the fading memory concept.

Now, we can turn on magnetic field diffusion models with memories.

\subsubsection{Fading memory in magnetic field diffusion}

The fading memory concept was touched earlier with equation (31). Actually, we immediately 
jumped to the model where instantaneous term (long time term) does not exist thus entering into the area supported by the concept of the Continuous Time Random Walk (CTRW) where long time term does not exist. This was done especially in order to demonstrate how time-fractional Caputo derivative can be implemented in a diffusion model with respect to the non-locality, i.e. the causality principle. Moreover, the models with the Caputo derivative are more familiar and the solutions developed can be easily understood. This models, could be applied (not in the scope of this work) to composite magnetic media where small magnetic particles (of nano or macro sizes) are dispersed (almost homogeneously) is non-magnetic matrix; the gaps between the magnetic kernels are zones with high resistances with respect to the magnetic field lines, such as gaps and obstacles in porous media where fractional modelling is widely applied.

However, let us consider the case where all terms of (31) take place. In the context of the magnetic field diffusion, this precisely means that after the initial relaxation and disappearance of the send term, there is continuous magnetic energy supply through the boundary $x=0$; the simple example is the Dirichlet problem. In such a case the complete model is

$$
\begin{gathered}
j_{B}(x, t)=-D_{B 0} \frac{\partial B}{\partial x}(x, t)- \\
-D_{B 1} \int_{-\infty}^{t} R(t-\tau) \frac{\partial B}{\partial x}(x, \tau) d \tau
\end{gathered}
$$

If the memory function is chosen to be singular power-law then the second term becomes the Riemann-Liouville fractional integral (34) and the flux-gradient relationship has be presented by an extended version of (35), namely

$$
j_{B}=-D_{B 0} \frac{\partial B}{\partial x}-D_{B 1} I_{t}^{\alpha}\left(\frac{\partial B}{\partial x}\right)
$$

After application of the continuity equation (32) we get

$$
\frac{\partial B}{\partial t}=D_{B 0} \frac{\partial^{2} B}{\partial x^{2}}+D_{B 1} I_{t}^{\alpha}\left(\frac{\partial^{2} B}{\partial x^{2}}\right)
$$

Here the non-locality is presented by the last term. This construction shows the main idea how non-locality has to be implemented at the level of constitutive equation. We will discuss a magnetic diffusion equation with exponential kernel next.

\subsubsection{Memory kernel effect on the fractional model}

Now, let us follows the main line drawn in the preceding point of this section and consider that flux gradient relationship contains all elements of the fading memory functional but now the convolution integral has exponential memory kernel, namely

$$
\begin{array}{r}
j_{B}=-D_{B 0} \int_{0}^{t} \delta_{D}(z) \frac{\partial B(x, z)}{\partial x} d z \\
-D_{B 1} \frac{1}{\tau} \int_{0}^{t} e^{-\frac{(t-z)}{\tau}} \frac{\partial B(x, z)}{\partial x} d z
\end{array}
$$

where the first term is the instantaneous one since the memory kernel is the Dirac delta $\delta_{D}$, while the second term has exponential memory as in the classical Cattaneo concept. This flux-gradient construction was investigated in [23] and resulted in a diffusion equation with a non-local damping term expressed through the Caputo-Fabrizio time-fractional derivative (78)

$$
\frac{\partial B}{\partial t}=D_{B 0} \frac{\partial^{2} B}{\partial x^{2}}+D_{B 1}(1-\alpha)^{C F} D_{t}^{\alpha}\left[\frac{\partial^{2} B}{\partial x^{2}}\right]
$$

solved in semi-infinite [25, 26] and finite domains [27.

In (77), the operator ${ }^{C F} D_{t}^{\alpha}$ is the Caputo-Fabrizio time fractional derivative of order $\alpha$ [24]

$$
\begin{array}{r}
C F D_{t}^{\alpha} B(x, t)= \\
\frac{M(\alpha)}{1-\alpha} \int_{0}^{t} \exp \left[-\frac{\alpha(t-s)}{1-\alpha}\right] \frac{d B(x, s)}{d t} d s
\end{array}
$$

and the relaxation time $\tau$ in (76) is related to the fractional order $\alpha$ as $\tau(0, \infty)=(1-\alpha) / \alpha$, $0<\alpha<1$ (see extended analysis in [28]). Moreover, the concept expressed by (76) is valid even in the case when the material exhibits spatial memory and leads to a spatial Caputo-Fabrizio derivative with exponential kernel [28,29]. It is obvious, that the non-locality is not lost despite the use of exponential kernel since the last term in (77) is responsible for this.

Similarly, any other relaxation functions invoked by the type of the relaxations in the observed physical problems, may form kernels of non-local 
terms, but this problem is more general and beyond the scope of this work (some examples are available in [30]).

\section{Conclusion}

This study addressed the magnetic field diffusion model solved in various situations by tools of fractional derivatives. The main results can be outlined as:

- The semi-derivative approach to the parabolic model (8) with Dirichlet boundary condition, and especially with time ramping (power-law) boundary condition allows a direct relation between the function and the gradient, and easy integration of the boundary condition. Moreover, the approximate integral-balance solution needs only a single integration step.

- The integral-balance method by the technology of double integration (DIM) allows straightforward approximate solutions of magnetic diffusion with field-dependent diffusivity (with negligible Joules effects, i.e unchanged material resistivity). The solutions, sharp and almost rectangular waves, are moving with finite speeds (due the degenerate nature of the model).

- The magnetic field diffusion with memory was demonstrated on the basis of a singular memory kernel (power-law allowing the Caputo time-fractional derivative to be applied. This fractionalization behaviour is analogue of the CTRW concept and allows easy the approximate integralbalance solutions to be applied.

- The fading memory approach and the causality principle were used to formulate a general approach to implement nonlocality in constitutive equation, and consequently to conservation laws; in the present case to the magnetic diffusion model.

- The problems and solutions presented demonstrate a variety of approaches where the fractional calculus can be applied efficiently for solving diffusion models, and particularly to the problems related to the magnetic field (straight lines) diffusion in ferromagnetic materials. This is only a step towards solutions of more complex problems and we see the use of the fractional calculus is promising.

\section{Acknowledgments}

The author thanks the editors of IJOCTA for possibility to create this special issue devoted to fractional calculus and publish results from my research program.

\section{References}

[1] Wilmot-Smith, A.L., Priest, E.R. \& Horning, G. (2005). Magnetic diffusion and the motion of field lines. Geophys. Astrophys.Fluid Dyn., 99(2), 177-197.

[2] Adam'yan, Yu.E., Vyrva, E.A., Krivosheev, S.I., \& Titkov, V.V. (2013). Diffusion of a pulsed magnetic field and electromagnetic forces in ferromagnets. Theor.Math. Phys., 53(10), 1397-1403.

[3] Schnitzer, O. (2014).Fast penetration of megagauss fields into metallic conductors. Phys. Plasmas, 21, 082306, doi $: 10.1063 / 1.4892398$.

[4] Xioa, B., Gu, Zh.W., Kan, M.X., Wang, G.H. \& Zhao, J.H. (2016). Sharp-front wave of strong magnetic field diffusion in solid metal. Phys. Plasmas, 23, 082104 doi : 10.1063/1.4960303.

[5] Yan, C., Xio, B., Wang.G.H., Jkan, M.X., Duan, S.C., Li, P. \& Sun, D. (2019).The second type of sharp-front wave mechanism of strong magnetic field diffusion in solid metal. AIP Advances, (9), 125008, doi : 10.1063/1.5124436.

[6] Chaikovsky, S.A., Oreshkin, V.I., Datsko, I.M., Labetskaya, N.L., Rybka, D.V. \& Ratakhin, N.A. (2015). Experimental study of the nonlinear diffusion of a magnetic field and skin explosion of cylindrical conductors. Phys.Plasmas, (22), 112704 doi : 10.1063/1.4935401.

[7] Agrawal, O.P. (2004). Application of fractional derivatives in thermal analysis of disk brakes, Nonlinear Dynamics, (38), 191-206.

[8] Kulish, V.V. \& Lage, J.L. (2000). Fractionaldiffusion solutions for transient local temperature and heat flux. J. Heat Transfer, 122(2), 372-376.

[9] Oldham, K.B. \& Spanier, J. (1974). The Fractional Calculus, Academic Press, New York, USA.

[10] Goodman, T.R. (1958). The heat balance integral and its application to problems involving a change of phase. Transactions of ASME, 80(1-2), 335-342. 
[11] Hristov, J. (2009). The heat-balance integral method by a parabolic profile with unspecified exponent:Analysis and Benchmark Exercises. Thermal Science, 33(2), 27-48.

[12] Hristov, J. (2016). An alternative integralbalance solution to transient diffusion of heat (mass) by time-fractional semiderivatives and semiintegrals: fixed boundary conditions. Thermal Science, 20(6), 1867-1878.

[13] Carslaw, H.S. \& Jaeger, J.C. (1959). Conduction of Heat in Solids, Oxford University Press, London.

[14] Hristov, J. (2017). Double integral-balance method to the fractional subdiffusion equation: approximate solutions, optimization problems to be resolved and numerical simulations. J. Vibration and Control, 23(7), $2795-2818$.

[15] Hristov, J. (2016). Integral solutions to transient nonlinear heat (mass) diffusion with a power-law diffusivity: a semi-infinite medium with fixed boundary conditions. Heat Mass Transfer, 52(3), 635-655.

[16] Hristov, J. (2018). Integral-balance solution to nonlinear subdiffusion equation, in Frontiers in Fractional Calculus, 2018, S. Bhalekar, Ed. Bentham Publ., Sharjah, vol.1, 171-106.

[17] Storm, M.L. (1951). Heat conduction in simple metals. J. Appl. Phys., 22(7), 940-951.

[18] Coleman, B. \& Noll, W. (1961). Foundations of Linear Viscoelasticity. Rev. Modern Phys., 33(2), 239-249.

[19] Coleman, B. \& Gurtin, M.E. (1967). Equipresence and constitutive equations for rigid heat conductors. Z.Angew.Math Phys., 18(3), 188-208.

[20] Gurtin, M.E. (1968). On the thermodynamics of materials with memory. Arch.Rational. Mech. Anal., 28(1), 40-50.

[21] Gurtin, M.E. \& Pipkin, A.C. (1968). A general theory of heat conduction with finite wave speeds. Arch.Rational Mech.Anal., 31(1), 113-126.

[22] Nussenzveig, H. (1972). Causality and dispersion relations, vol.95 of Mathematics in science and engineering, Academic Press, NY.

[23] Hristov, J. (2016). Transient heat diffusion with a non-singular fading memory:from the Cattaneo constitutive equation with Jeffrey's kernel to the Caputo-Fabrizio time-fractional derivative. Thermal Science, 20(2), 765-770.
[24] Caputo, M. \& Fabrizio, M. (2015). A new definition of fractional derivative without singular kernel. Progr. Fract. Differ. Appl., 1(1), 73-85.

[25] Avci, D., Yavuz, M. \& Ozdemir, N. (2019). The fundamental solutions to Cauchy and Dirichlet problems for heat conduction equation with Caputo-Fabrizio operator. Chapter 4, In: Heat Conduction,J. Hristov and R. Bennacer,(eds), NOVA Sci. Publ., 95-107.

[26] Sene, N. (2019). Analytical solutions of Hristov diffusion equations with non-singular fractional derivatives. Chaos, 29, 023112, doi : $10.1063 / 1.5082645$.

[27] Iskender Eroglu, B.B. \& Avci, D. (2021). Separable solutions of Cattaneo-Hristov heat diffusion equation in a line segment: Cauchy and source problems. Alexandria Engineering Journal, 60(2), 2347-2353.

[28] Hristov, J. (2018). Derivatives with nonsingular kernels from the Caputo-Fabrizio definition and beyond: Appraising analysis with emphasis on diffusion models, In:Frontiers in Fractional Calculus: Chapter10, Bentham Science Publishers, 270-342.

[29] Hristov, J. (2017). Steady-state heat conduction in a medium with spatial non-singular fading memory: derivation of CaputoFabrizio space-fractional derivative with Jeffrey's kernel and analytical solutions Thermal Science, 21(2), 827-839.

[30] Hristov J. (2019). Bio-heat models revisited: concepts, derivations, nondimensalization and fractionalization approaches. Frontiers in Physics, 21 (November) 2019, doi : 10.3389/fphy.2019.001897: 189.

Jordan Hristov is a Professor of Chemical Engineering. His areas of interest are Transport Phenomena, Mathematical Modelling, Fractional Calculus, Heat Transfer, Approximate analytical methods, Dimensional analysis. With 185 research papers in various journals of high repute and listed by Web of Science he got total citations more than 2150 with hindex of 22 (Web of Science). In 2020 he was enlisted in the Stanford list of highly cited scholars among the first percent (at 318 position in Chemical Engineering). Professor Jordan Hristov is a Member of Editorial Boards of: Thermal Science, Particuology, Fractal and Fractional, Progress in Fractional Differentiations and Applications. The principle direction of his research in the last decade is related to approximate analytical solutions of non-linear and fractional problems, applications of non-singular fractional operator to non-local problems, fractional viscoelasticity, heat conduction, non-linear diffusion.

(1) https://orcid.org/0000-0002-7957-8192 
An International Journal of Optimization and Control: Theories \& Applications (http://ijocta.balikesir.edu.tr)

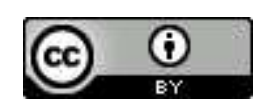

This work is licensed under a Creative Commons Attribution 4.0 International License. The authors retain ownership of the copyright for their article, but they allow anyone to download, reuse, reprint, modify, distribute, and/or copy articles in IJOCTA, so long as the original authors and source are credited. To see the complete license contents, please visit http://creativecommons.org/licenses/by/4.0/. 